\title{
Möglichkeiten der Nutzung von Media-Analyse- Fernsehdaten für Sekundäranalysen von 1972 bis heute
}

\author{
Jörg Hagenah
}

Nachdem in MEK 3/2006 die Nutzungsmöglichkeiten der Media-Analyse-Radiodaten im Fokus standen, beinhaltet dieser zweite Teil eine Dokumentenanalyse der Fragebögen und Codepläne zur Fernseh-Abfrage. Problemlos lassen sich die bis 1996 bzw. 1999 erhobenen senderspezifischen Abfrageblöcke zu General-und Zeitfilter längsschnittlich nutzen. Eingeschränkt ist jedoch die longitudinale Nutzbarkeit von Frequenzabfrage und Tagesablauf, die erst seit 1987 den gesamten Tag und nicht nur die Zeit zwischen 17.00 und $20.00 \mathrm{Ubr}$ umfassen. Darüber hinaus werden pauschale TV-Daten zur wöchentlichen Fernsebnutzung innerhalb der Freizeit und im Tagesablauf abgefragt.

Schlagwörter: Fernsehen, Sekundäranalyse, Longitudinalforschung, Mediennutzung, Methoden

Nachdem in M\&K 3/2006 in einem ersten Teil die Nutzungsmöglichkeiten der Media-Analyse-Radiodaten im Mittelpunkt standen (Hagenah, 2006), sollen hier im Fokus einer analogen Dokumentenanalyse die Media-Analyse-Fernsehdaten stehen. Ziel des Beitrags ist es, auch für die Abfrage der TV-Nutzung einen detaillierten Überblick darüber zu verschaffen, wann welche Variablen auf welche Weise erhoben wurden. Somit kann die Sekundäranalyse aus unterschiedlichsten Perspektiven vorbereitet, die Dokumentation der Aufbereitungsarbeiten sichergestellt und die Mediaforschungspraxis transparent dargestellt werden.

Bezüglich der Erbebungs- und Variablenstruktur der Media-Analysen (MA) kann auf den vorhergehenden Beitrag verwiesen werden (Hagenah, 2006: 459-461). Den größten Einfluss auf die Variablenstruktur der Fernsehvariablen hatten - wie im Folgenden noch ausführlicher gezeigt wird - erstens die 1987 erfolgte Umstellung der Single-Source-Studie ${ }^{1}$ auf eine nach Medienarten getrennte Abfrage und zweitens die schrittweise Herausnahme der senderspezifischen TV-Abfrage von 1997 bis 2000 . Zweiteres sei nach Müller als Folge der Ergebnisse der Methodentests der AG.MA 1995 anzusehen, da das gelegentlich unbewusst und häufig nebenbei gehörte Medium Radio besser und vollständiger erinnert werde, wenn die Befragten nicht durch eine differenzierte Abfrage der Fernsehnutzung abgelenkt werden (Müller, 2003: 55). Beide Umbrüche betreffen sowohl originäre wie abgeleitete Variablenkomplexe. Gegenstand dieses Beitrages sind die originären Variablen; auf die abgeleiteten (Zusammenfassungen, Nutzungswahrscheinlichkeiten, Kontaktsummen, Varianzen; siehe Hagenah, 2006: 477-480) wird nicht weiter eingegangen.

1 Insbesondere in den 1970er-Jahren wurde synonym auch der Begriff Multi-Media-Analyse verwendet. 


\section{Möglichkeiten der Nutzung von Fernsehdaten}

Als die wichtigste Quelle für Fernsehdaten muss zweifellos der im Auftrag der Arbeitsgemeinschaft Fernsehforschung (AGF) von der Gesellschaft für Konsumforschung (GfK) telemetrisch in einem Panel erhobene AGF/GfK-Datensatz bezeichnet werden (www.gfk.de, 2006). Daher ist er auch Bestandteil der wichtigsten Werbeplanungsquelle (Müller \& Mai, 2006: 21 f.) - der so genannten Intermedia-Datei der Media-Analyse, die per Fusion Nutzungsdaten zu allen relevanten Medienarten enthält (Mai, 2003). Allerdings ist der AGF/GfK-Paneldatensatz methodenbedingt mit Problemen der Gewöhnung (dem sog. „Panel-Effekt“; siehe Bortz \& Döring, 2002: 260, 450 f.) und vor allen Dingen mit der im Folgenden behandelten Repräsentativität behaftet:

1. Aufgrund der hohen Erhebungskosten beschränkt sich die Stichprobe auf eine im Vergleich zur MA Radio mit über 50.000 Befragten etwas kleinere Stichprobe mit fast 13.000 Personen (www.agf.de, 2006), somit sind der Zielgruppenanalyse oder vielmehr der Sub-Zielgruppenanalyse deutlichere natürliche Grenzen gesetzt.

2. Wie alle Panelerhebungen (Diekmann, 2004: $271 \mathrm{f}$.) hat auch die AGF-GfK-Forschung mit dem Problem der Panel-Mortalität zu kämpfen ${ }^{2}$.

Verstärkt werden diese datensatzbezogenen Argumente durch zwei pragmatische Gesichtspunkte. Erstens liegen die Paneldaten bisher nicht als Individualdatensätze in SPSSForm vor; eine entsprechende Datenaufbereitung ist zwar technisch möglich, aber problematisch und sehr aufwändig (Karnowski 2003: 59 ff.; Bilandzic 1998: 748). Zweitens sind die Datensätze nicht im Zentralarchiv für empirische Sozialforschung gespeichert und können nur in Kooperation mit einem der Mitglieder der AGF genutzt werden.

Wenn die messtechnischen Vorteile des telemetrischen Verfahrens außer Acht gelassen werden, erscheint es mit Blick auf die Kriterien Repräsentativität und Teilpublika-Analysepotenzial für bestimmte longitudinale Untersuchungszecke sinnvoller, die Daten der Media-Analyse zu verwenden oder zumindest zu Validierungszwecken hinzuzuziehen. Durch die Konvertierung der MA-Daten in SPSS (Hagenah, Meulemann \& Akinci 2006) existieren nutzbare Individualdatensätze, die den Einsatz von unterschiedlichsten statistischen Verfahren bei der Sekundäranalyse ermöglichen. Einen ersten Gesamtdatensatz mit den aus 27 Querschnitten von 1977 bis 2004 zusammengefügten Dateien haben Best und Hagenah (2006a) erstellt. Er enthält zwar nur wenige Variablen (pauschale Fernsehnutzung des Vorabendprogrammes im Tagesablauf sowie Angaben zu Geschlecht, Bildung und Alter), aber 825.036 Fälle. Der exemplarisch aufbereitete Datensatz ermöglicht zwar keine senderspezifische Unterteilung - hierfür müssten die AGF/ GfK-Daten bzw. deren Vorgänger-Datensätze genutzt werden - aber eine detaillierte Untersuchung von TV-Teilpublika über einen langen Zeitraum.

2 Das Problem der Panel-Mortalität versucht man auszugleichen, indem auf Basis der Erhebungskennziffern der Media-Analysen die kleiner gewordenen Zellen mit neu zu rekrutierenden Personen oder Haushalten aufgefüllt werden (www.agf.de, 2006). Auf diese Weise repräsentiert die Stichprobe zwar wieder einigermaßen die Grundgesamtheit für das aktuelle Erhebungsjahr. Allerdings muss man sich somit der eigentlichen Stärke von Paneldaten berauben, nämlich der Längsschnittanalyse auf der Individualdatenebene. Wie eine Untersuchung von Karnowski (2003: 66) zeigte, waren von den etwa 8.000 Panelpersonen des Jahres 1994 nur noch etwa 850 Personen in der im Jahr 2001 immerhin 11.000 Personen umfassenden Stichprobe enthalten. Wenn man also AGF/GfK-Daten über einen längeren Zeitraum betrachtet, können Sekundäranalysen nur zu einem sehr geringen Anteil eine Veränderungsdynamik auf der individuellen Ebene nachzeichnen. Zum größten Teil erlauben eben auch derartige Paneldaten - bei einer Berücksichtigung der vollen Stichproben - faktisch lediglich Trendanalysen. 
Praktische Voraussetzung für längsschnittliche Analysen ist, dass in den Erhebungen über die Zeit vergleichbare Variablen zu finden sind, was bisher noch nicht näher untersucht wurde.

Ziel dieses Beitrags ist es daher, für die Abfragen zur Fernsehnutzung einen detaillierten Überblick darüber zu verschaffen, welche Variablen für eine längsschnittliche Nutzung zur Verfügung stehen. Als Informationsbasis für eine Dokumentenanalyse dienen prinzipiell die MA-Codepläne und -Fragebögen der Jahre 1972 bis 2003; diese beschränken sich allerdings bei den älteren Dokumenten auf die Zeit ab 1976, da die ersten Jahrgänge entweder nur rudimentär aufbereitet vorliegen oder lückenhaft sind. Da in dem ersten Artikel zu den Radiodaten (Hagenah, 2006) sehr detailliert Ausschnitte aus Fragebögen und Codeplänen in Abbildungen gezeigt wurden, wird an dieser Stelle auf eine Wiederholung von sehr ähnlichen Abbildungen verzichtet. Stattdessen wird der genaue Wortlaut der Abfragen und Antwortkategorien wiedergegeben und ein Verweis auf die entsprechenden Abbildungen des ersten Teiles angeführt.

\section{Fernsehabfragemodelle von $\mathbf{1 9 7 2}$ bis heute}

Wie die nachfolgende Dokumentenanalyse im Detail zeigen wird, lässt sich die Fernsehabfrage in zwei Epochen ${ }^{3}$ teilen. Die erste Epoche beinhaltet mehrere Phasen einer senderspezifischen Fernsehabfrage nach dem Muster der Standard-MA-Abfrage in der Zeit von 1972 bis 1999, die zweite Epoche beinhaltet seit 2000 bis heute mehrere Phasen einer pauschalen oder senderunspezifischen Fernsebabfrage.

\subsection{Senderspezifische Fernsehabfragen von 1976 bis 1999}

Die Untersuchungsanlage der senderspezifischen Standard-MA-Abfrage zum Fernsehen ist vom Grundprinzip her aufgebaut wie die Radioabfrage der MA. Dem Generalfilter folgen der Zeitfilter, die Frequenzabfrage (Sehhäufigkeit pro Woche) und die Fragen zum Tagesablauf (siehe Mai, 2003). Den Fragebögen und Codeplänen lässt sich entnehmen, dass diese komplexe Form mit den vier Standardschritten lediglich von 1987 bis 1996 eingesetzt wurde. Von 1997 bis 1999 wurden nur noch zwei der vier Abfrageblöcke erhoben (Generalfilter, Frequenzabfrage) und seit der Erhebungsumstellung vom persönlichen zum telefonischen Interview im Jahr 2000 gibt es keine senderspezifischen Fernsehabfragen mehr.

Mit Hilfe der Dokumentenanalyse lässt sich das ältere Abfragemodell der Zeit von $1972^{4}$ bis 1986 rekonstruieren. Es ähnelt der Standard-MA-Abfrage und hatte dasselbe Grundprinzip, dennoch lassen sich neben Gemeinsamkeiten auch ein paar Unterschiede bei Frequenz- und Tagesablaufabfrage konstatieren, auf die in den entsprechenden Unterabschnitten genauer eingegangen wird.

Die einzelnen Kategorien der Erhebungsmodelle werden in der genannten Reihenfolge näher vorgestellt, indem für jede Variante die jeweils jüngsten Abfrage- und Kodierungsmodelle dargestellt werden.

3 Theoretisch ließen sich auch drei Epochen benennen: Zwischen 1997 und 1999 gab es eine Umbruchphase, bei der die senderspezifischen Fernsehabfragen stufenweise herausgenommen wurden.

4 Dies gilt nur eingeschränkt für die Jahre 1972 bis 1975, da während der Umbauphase noch experimentiert wurde. Nach Scheler (1983:387) seien „mit MA 76 die wesentlichen Probleme des Ausbaus zur Multi-Media-Analyse gelöst“" worden. Daher wird als älteste MA grundsätzlich diejenige des Jahres 1976 aufgeführt. 


\subsubsection{Generalfilter}

Seit 1970 verwendeten die Leser-Analyse ( $L A)$ und deren Nachfolger die $M A$ in allen Erhebungen einen Generalfilter, durch den sich die Befragten als Nutzer eines Mediums „qualifizieren“ (vgl. Koschnick, 2004a). In diesem ersten Komplex wird danach gefragt, von welchen Sendern schon Sendungen gesehen wurden. Eingesetzt wurde der TV-Generalfilter letztmalig im Jahr 1999.

Die im Fragebogen enthaltenen Intervieweranweisungen und Fragen sahen von 1976 bis 1999 wie folgt aus (z. B. ma_96_EM_frb.pdf, S. 78 f. ${ }^{5}$, Hagenah, 2006: 463):

\section{INT: Fernsehkarten aus dem Umschlag „Fernsehen“ herausnehmen}

Blätter 4R, 4L aufschlagen

Auf diesen Karten sind Erkennungsfiguren für die verschiedenen Fernsehsender und Fernsehprogramme abgebildet. Von welchen Sendern haben Sie schon Sendungen gesehen?

INT: Fernseh-Karten übergeben. Vom Befragten auf die Blätter 4R und 4L sortieren lassen.

Antworten im Schema ankreuzen. Falls keinen Sender „schon gesehen“, weiter mit Frage 7.

$[4 R=$ Von diesem Sender habe ich noch nie Sendungen geseben, $4 L=$ Von diesen Sendern habe ich schon Sendungen gesehen; ma_96_em_frb.pdf, S. $141 \mathrm{f}$.]

Um den Befragten die Erinnerung zu erleichtern und um den Befragungsprozess zu beschleunigen, wurden in allen senderspezifischen Fernsehabfragen so genannte „Titelkarten“ eingesetzt (aided recall). Für jeden abgefragten Fernsehsender existierte eine Karte, die den Sendernamen, das Logo und bei öffentlich-rechtlichen Sendern die so genannten „Werbe“-Erkennungsfiguren, wie die „Mainzelmännchen“ beim ZDF oder „Antje das Walroß“ vom ARD-Sender NDR, enthält (z. B. ma_99_EM_frb.pdf, S. 60, 61, 88-95). Die Befragten wurden nun gebeten, die Karten zu sortieren und entsprechend der Antwortmöglichkeiten zwei „Häufchen“ zu bilden. Die Karten von dem Häufchen „von diesen Sendern habe ich noch nie Sendungen gesehen " wurden sofort weggesteckt, diejenigen von dem Häufchen „von diesen Sendern habe ich schon Sendungen gesehen“ wurden im Fragebogen als solche angekreuzt. Zu diesen Fernsehsendern wurde im Anschluss die Folgefrage (Zeitfilter) gestellt.

Codiert wurden die aus der Generalfilter-Frage gewonnenen Informationen von 1976 bis 1996 zusammen mit den Informationen aus der nachfolgenden Zeitfilterfrage. Unter den in den Codeplänen genannten Feldnummern befinden sich daher in den Datensätzen unter dem Oberbegriff „Zeitfilter“ (siehe Abschnitt 2.1.2) sowohl die Daten aus der Generalfilterfrage als auch die Informationen, die aus der im engeren Sinne Zeitfilter genannten Frage stammen (z. B. ma_96_EM_cdb.pdf, S. 128., vgl. Hagenah, 2006: 463). Die Ausprägungen 1 bis 3 bei der Zeitfilterkodierung können zusammengenommen auch als Antwortmöglichkeit „schon mal gesehen“ zur Generalfilterfrage gewertet werden. „6 = noch nie gesehen “ und „, $5=$ keine Angabe“ stammen direkt aus der Generalfilterfrage.

5 Alle beschriebenen Beispiele können auch in den Fragebögen leicht nachgeschaut werden, da sich diese zum freien Download auf der Seite www.wiso.uni-koeln.de/medien/ befinden. Alle Seiten-Angaben zu Fragebögen und Codeplänen beziehen sich auf die Seitenangaben im dazugehörigen PDF-Dokument.. 
In den Datensätzen 1997 bis 1999 befinden sich unter dem Oberbegriff „Zeitfilter“ (z. B. ma_99_EM_frb.pdf, S. 18 f.) nur noch die Generalfilterinformationen „1 = schon gesehen“, „, 2 = noch nie gesehen“ und „, $3=$ keine Angabe“. Der Abfrageblock „Zeitfilter" war nicht mehr Teil des Erhebungsprogramms, wie auch im folgenden Abschnitt näher erläutert wird.

Welche Einzelsender von wann bis wann Teil der Erhebungen waren, lässt sich Tabelle 1 entnehmen. Insbesondere für die „Regionalfenster“ der ARD und für das ZDF können lange Zeitreihen von 1976 bis 1999 erstellt werden. Die ersten Privatsender wurden 1986 (RTL) und 1987 (Sat.1) abgefragt. Diese Erhebungsinformationen gelten sowohl für die Generalfilter- als auch für die nachfolgend ausführlicher beschriebene Zeitfilterabfrage. $^{6}$

Tabelle 1: Ausschnitt aus der MA-Variablenübersichtstabelle madatsyn 1.0_short $($ General -+ Zeitfilterabfrage $=$ Zeitfilter $)$

\section{Variablenname}

Zeilennr. Identifikationsmerkmale

Erbebungszuordnung
Erhebungs- Erhebungszeitraum anzahl

insgesamt

Erste Erhebung Erhebung

\begin{tabular}{llrrr}
\hline 31046 & Zeitfilter pro Sender(Fernsehen) & & & \\
31048 & Das erste deutsche Fernsehen (ARD) & 14 & MA 87 EM & MA 99 EM \\
31049 & Sender Freies Berlin (SFB) / ORB & 25 & MA 76 & MA 99 EM \\
31050 & Radio Bremen & 19 & MA 82 & MA 99 EM \\
31051 & Norddeutsches Fernsehen & 25 & MA 76 & MA 99 EM \\
31052 & Westdeutsches Fernsehen & 25 & MA 76 & MA 99 EM \\
31053 & Hessisches Fernsehen & 25 & MA 76 & MA 99 EM \\
31054 & Saarländisches Fernsehen & 25 & MA 76 & MA 99 EM \\
31055 & Südwest und Süddeutsches Fernsehen & 25 & MA 76 & MA 99 EM \\
31056 & Bayerisches Fernsehen & 25 & MA 76 & MA 99 EM \\
31057 & Drittes Fernsehprogramm ORB & 9 & MA 92 EM & MA 99 EM \\
31058 & Drittes Fernsehprogramm MDR & 9 & MA 92 EM & MA 99 EM \\
31059 & Zweites deutsches Fernsehen (ZDF) & 24 & MA 76 & MA 99 EM \\
31060 & Drittes Fernsehprogramm HR (Hessen 3) & 13 & MA 87 EM & MA 99 EM \\
31061 & Pro 7 & 10 & MA 91 EM & MA 99 EM \\
31062 & RTL Television & 15 & MA 86 & MA 99 EM \\
31063 & Sat.1 & 14 & MA 87 EM & MA 99 EM \\
31064 & Tele 5 & 2 & MA 91 EM & MA 92 EM \\
31065 & Alle Sender & 17 & MA 78 & MA 99 EM \\
31066 & 3 Sat & 1 & MA 87 EM & MA 87 EM \\
31067 & Drittes Fernsehprogramm BR (Bay.fern- & 8 & MA 87 EM & MA 99 EM \\
& sehen) & &
\end{tabular}

6 Wie in den Codeplänen sind auch in der Tabelle „madatsyn 1.0“ die Informationen zu Generalfilter- und Zeitfilterabfragen unter dem Oberbegriff „Zeitfilter“ enthalten. 
Fortsetzung Tabelle 1

\begin{tabular}{|c|c|c|c|c|}
\hline \multirow{3}{*}{ Zeilennr. } & \multicolumn{4}{|l|}{ Variablenname } \\
\hline & \multirow{2}{*}{$\begin{array}{l}\text { Identifikationsmerkmale } \\
\text { Erbebungszuordnung }\end{array}$} & \multirow{2}{*}{$\begin{array}{l}\text { Erhebungs- } \\
\text { anzahl } \\
\text { insgesamt }\end{array}$} & \multicolumn{2}{|c|}{ Erhebungszeitraum } \\
\hline & & & $\begin{array}{l}\text { Erste } \\
\text { Erhebung }\end{array}$ & $\begin{array}{l}\text { Letzte } \\
\text { Erhebung }\end{array}$ \\
\hline 31068 & Drittes Fernsehprogramm NDR / RB (N3) & 8 & MA 87 EM & MA $99 \mathrm{EM}$ \\
\hline 31069 & Drittes Fernsehprogramm SFB B1 & 7 & MA 94 EM & MA $99 \mathrm{EM}$ \\
\hline 31070 & $\begin{array}{l}\text { Drittes Fernsehprogramm SR / SDR / SWF } \\
\text { (Südwest3) }\end{array}$ & 8 & MA 87 EM & MA 99 EM \\
\hline 31071 & Drittes Fernsehprogramm WDR (WDF) & 8 & MA 87 EM & MA $99 \mathrm{EM}$ \\
\hline 31072 & DSF Deutsches Sportfernsehen & 7 & MA 94 EM & MA 99 EM \\
\hline 31073 & Kabelkanal & 7 & MA 94 EM & MA $99 \mathrm{EM}$ \\
\hline 31074 & Mitteldeutscher Rundfunk (MDR) & 7 & MA 94 EM & MA 99 EM \\
\hline 31075 & $\mathrm{n}-\mathrm{tv}$ & 7 & MA 94 EM & MA 99 EM \\
\hline 31076 & RTL 2 & 7 & MA 94 EM & MA 99 EM \\
\hline 31077 & Sky Channel & 1 & MA 87 EM & MA 87 EM \\
\hline 31078 & VOX & 7 & MA 94 EM & MA 99 EM \\
\hline 31079 & sonstige Fernsehprogramme & 8 & MA 87 EM & MA 99 EM \\
\hline 31080 & Dritte Programme & 10 & MA 76 & MA 86 \\
\hline 31081 & Super RTL & 5 & MA 96 EM & MA 99 EM \\
\hline 31082 & $\operatorname{tm} 3$ & 4 & MA 97 EM & MA 99 EM \\
\hline
\end{tabular}

Anmerkung: EM = Elektronische Medien (auch Elektronische Tranche oder seit 1997 MA Radio genannt); die Zeilennummer entspricht derjenigen der madatsyn 1.0, die die jeweilige Feldnummer pro Erhebung von 1972 bis 2000 enthält, also den Speicherort in den SPSS-Datensätzen.

\subsubsection{Zeitfilter}

Für alle „schon mal gesehenen Sender“ wurde von 1976 bis $1996^{7}$ im so genannten Zeitfilter ermittelt, wann zuletzt eine Sendung von einem TV-Sender gesehen wurde. Die im Fragebogen enthaltenen Intervieweranweisungen und Fragen sahen wie folgt aus (z. B. ma_96_EM_frb.pdf, S.78 f.; vgl. Hagenah, 2006: 465):

INT: Frage 5 für alle lt. Frage 4 „schon gesehenen“ Sender stellen.

\section{Blatt 5 aufschlagen.}

Wann haben Sie zuletzt eine Sendung von gesehen?

7 Laut Codeplan MA 97 (ma_97_EM_cdb.pdf, S. 114) sollte es auch in der MA 1997 Informationen zum Zeitfilter geben. Aber weder im dazugehörigen Fragebogen noch im Datensatz lassen sie sich finden. Offensichtlich enthält der Codeplan fälschlicherweise die Codierung des Vorjahres. Eine Häufigkeitszählung zeigt, dass sich der Datensatz auf die Generalfilterinformationen beschränkt. 
Hier habe ich eine Zeiteinteilung, die Ihnen vielleicht helfen kann, die richtige Antwort zu finden.

INT: Fernseh-Karten einzeln nacheinander auf Blatt 5A vorlegen. Antworten im Schema ankreuzen.

[Blatt 5: innerhalb der letzten 2 Wochen/2-4 Wochen her/länger her; Blatt $5 A$ ist leer, enthält lediglich einen Rabmen für die Karten, ma_96_em_frb.pdf, S. 143 f.]

Codiert wurden die Antworten zusammen mit den Generalfilterdaten mit $1=$ innerhalb der letzten 2 Wochen, 2 = 2 bis 4 Wochen her, 3 = länger her, 4 = keine Angabe (aus Zeitfilter), $5=$ keine Angabe (aus Generalfilter) und $6=$ noch nie gesehen (z. B. ma_96_EM_cdb.pdf, S. 128; vgl. Hagenah, 2006: 465)

\subsubsection{Frequenzabfrage: Sehbäufigkeit (pro Zeitabschnitt) pro Sender}

In der dritten Stufe „Sehhäufigkeit pro Sender“ wurde von 1987 bis 1999 ermittelt, an wie vielen Werktagen von Montag bis Samstag jeder einzelne Sender gesehen wurde. Von 1984 bis 1986 - also in den letzten drei „Single-Source-Studien“ - ist vermutlich aus (zeit-)ökonomischen Gründen kein entsprechender Abfrageblock eingesetzt worden. Von 1976 bis 1983 wurden die Informationen zur Sehhäufigkeit detaillierter pro (Tages-) Zeitabschnitt (bspw. 17.00 bis 17.30 Uhr) und Sender erhoben. Auf die letztgenannte Erhebungsform und deren Kompatibilität zur jüngeren Abfrage wird nachher gesondert eingegangen.

Zur „Sehhäufigkeit pro Sender“ wurden von 1987 bis 1999 alle Personen befragt, die Sendungen des Einzelsenders innerhalb der letzten zwei Wochen gesehen haben ${ }^{8}$. Die im Fragebogen enthaltenen Intervieweranweisungen und Fragen sahen wie folgt aus (z. B. ma_99_EM_frb.pdf, S. 18 f., vgl. Hagenah, 2006: 466):

\section{INT: Frage 31 für alle lt. Frage 30 „schon gesehenen“ Sender stellen.}

\section{Blatt 31 aufschlagen.}

Wenn Sie an eine normale Woche in der letzten Zeit denken: An wievielen von den üblichen 6 Werktagen von montags bis samstags sehen Sie im allgemeinen Sendungen vom

Und wie ist es mit Sendungen vom

INT: Fernsehkarten einzeln nacheinander auf Blatt $31 \mathrm{~A}$ vorlegen.

Anzahl der genannten Werktage (1 bis 6) im betreffenden Kästchen eintragen.

Falls ein Sender „so gut wie nie“ gesehen wird, eine Null (=0) eintragen.

[Blatt 31: An den 6 Wochentagen Montag bis Samstag sehe ich im allgemeinen Sendungen vom : an 6 Tagen = an jedem Tag; an 5 Tagen; an 4 Tagen; an 3 Tagen; an 2 Tagen; an 1 Tag;

0 nie = an keinem Tag; Blatt 31A ist leer; ma_99_em_frb.pdf, S. 63]

8 Da zwischen 1997 und 1999 keine Zeitfilterangaben erhoben wurden, wurden in diesem Zeitraum alle Personen, die schon mal Sendungen des Senders gesehen haben (Generalfilter) zur „Sehhäufigkeit“ befragt. 
Codiert wurden die Informationen von „0 $=0$ von 6 Werktagen“ bis „6= 6 von 6 Werktagen"9 (ma_99_EM_cdb.pdf, S. 86).

Von 1976 bis 1983 wurde die Sehhäufigkeit detaillierter pro Zeitabschnitt und Sender abgefragt. Folgender Unterschied ist im Vergleich zwischen den Variablen Sehhäufigkeit pro Zeitabschnitt und (Woche pro) Sender (1976 bis 1983) und Sehhäufigkeit (pro Woche) pro Sender (ab 1987) erkennbar: Vor 1987 wurden alle Personen aus dem weitesten Seherkreis (WSK) zu sechs halbstündigen Zeitabschnitten zwischen $17.00 \mathrm{Uhr}$ und 20.00 Uhr und pauschal für die Zeit vor 17.00 Uhr gefragt, an wie vielen Werktagen in einer normalen Woche die (öffentlich-rechtlichen) Einzelsender gesehen wurden.

Die im Fragebogen enthaltenen Intervieweranweisungen und Fragen sahen für die Sebhäufigkeit pro Zeitabschnitt pro Sender wie folgt aus (ma_83_frb.pdf, S. 21 f., vgl. Hagenah, 2006: 468):

\section{INT: Blatt 36 aufschlagen:}

\section{Falls „innerhalb der letzten 2 Wochen“ zu einer Zeit ferngesehen wurde:}

Wenn Sie an eine normale Woche in der letzten Zeit denken. An wievielen von den 6 Werktagen montags bis samstags hören Sie im allgemeinen in der Zeit vor/ zwischen ... Uhr und ... Uhr den ....?

Und wie ist es mit dem ...?

INT: Mit der bei Frage 35 zuerst genannten Zeit beginnen und Fernseh-Karten einzeln nacheinander auf Blatt 36A vorlegen! Alle bei Frage 35 genannten Zeiten durchgehen und für jede Zeit Fernseh-Karten einzeln nacheinander auf Blatt 36A vorlegen!

Zahl der genannten Werktage (1 bis 6) im betreffenden Kästchen eintragen.

Falls ein Sender zu einer Zeit „nie“ gesehen wird, eine Null (=0) eintragen.

Weiter mit Frage 37 auf der Rückseite des Kästchen-Schemas!

[Blatt 36: In einer normalen Woche sehe ich im allgemeinen in der Zeit zwischen und Uhr vom Sender Fernseben: an 6 Tagen = an jedem Tag; an 5 Tagen; an 4 Tagen; an 3 Tagen; an 2 Tagen; an 1 Tag; 0 nie = an keinem Tag; Blatt 36A ist leer; ma_83_frb.pdf, S. 29f.]

Vom Interviewer wurden daraufhin die entsprechenden Antworten von „ $0=$ nie zu dieser Zeit" bis „6 = an allen Werktagen“ für jeden Zeitabschnitt in den Fragebogen eingetragen. Beispielsweise wurde für die Zeit zwischen 17.00 Uhr und 17.30 Uhr die Anzahl der Sehtage in einer normalen Woche ermittelt ${ }^{10}$. Seit 1987 spielen diese Zeitabschnitte keine Rolle mehr, und es wird nur noch nach der Sehhäufigkeit pro Woche gefragt.

Es gilt zu prüfen, ob die entsprechenden Daten beider Fragevarianten miteinander verknüpft werden können: Es ist annäherungsweise möglich, die älteren Daten in das schmalere Korsett der aktuelleren Erhebungen zu zwängen. Dazu kann bei den Daten von 1976 bis 1983 der maximale Tagewert aus den Zeitabschnittswerten herangezogen werden. Es ist jedoch davon auszugehen, dass dieser aus zwei Gründen eher zu klein sein wird:

9 Zusätzlich wurde „7 = keine Angabe“ codiert, außerdem enthalten ist die Generalfilterinformation „8 = nicht gesehen“.

10 Zusätzlich codiert wurden jeweils $7=$ keine Angabe und $8=$ nicht WSK (Weitester Seherkreis). 
Erstens wird die Fernsehnutzung zur Hauptzeit nach 20.00 Uhr vernachlässigt. So würde es passieren, dass beispielsweise berufstätige Vielseher, die erst nach $20.00 \mathrm{Uhr}$ nach Hause kommen und dann aber regelmäßig bis zum Sendeschluss vorm Fernseher sitzen, als Wenigseher mit 0 Tagen zu den erfassten Zeitabschnitten gewertet würden.

Die zweite Problematik soll anhand einer Tabelle mit konstruierten Nutzerdaten für das ZDF demonstriert werden (Tabelle 2). Angenommen, jemand sieht in einer normalen Woche von Montag bis Samstag regelmäßig zwischen $17.00 \mathrm{Uhr}$ und 17.30 das ZDF, so würde das in der Wochenstatistik wie bei Person 1 dargestellt aussehen. Der maximale Wert pro Zeitabschnitt wäre 6 und würde der tatsächlichen Sehhäufigkeit pro Sender in einer normalen Woche entsprechen. Sollte dagegen jemand, der von Montag bis Freitag sogar durchgehend von 17.00 bis 18.30 Uhr ZDF konsumiert und am Samstag zwischen 17.00 und $18.00 \mathrm{Uhr}$ gar nicht und um 18.00 bis $18.30 \mathrm{Uhr}$ die ARD-Sportschau schauen und erst wieder um 19.30 Uhr seinen Stammsender ZDF einschalten ${ }^{11}$, würde der maximale Zeitabschnittswert für die Person nur noch bei 5 liegen und somit kleiner sein als der reale Wert 6 Tage. Der eigentliche Wert 6 kann aber deshalb nicht ermittelt werden, weil - abweichend zu dem konstruierten Wochenmodell in Tabelle 1 - bei dem älteren Modell keine Angaben zu den einzelnen Wochentagen, sondern nur zu einer „normalen Woche" vorliegen. Letztlich ist es aber möglich, die Daten vor und ab 1987 in eine Variable zu „zwängen“, der beschriebene Methodeneffekt sollte aber beachtet werden. Somit stehen Frequenzdaten - mit einer kleinen Lücke zwischen 1984 bis 1986 - von 1976 bis 1999 zu Verfügung.

Tabelle 2: Konstruiertes Wochenmodell zur Erklärung des Methodeneffekts bei der Umkodierung der maximalen Zeitabschnittsdaten zur Sebhäufigkeit pro Woche: Beispiel ZDF

\begin{tabular}{|c|c|c|c|c|c|c|c|c|c|}
\hline & & Mo & $\mathrm{Di}$ & $\mathrm{Mi}$ & Do & $\mathrm{Fr}$ & Sa & $\begin{array}{l}\text { Max aus pro } \\
\text { Zeitabschnitt }\end{array}$ & $\begin{array}{l}\text { Sehhäufigkeit } \\
\text { pro Sender }\end{array}$ \\
\hline \multirow[t]{4}{*}{ Person 1} & $17.00-17.30$ & 1 & 1 & 1 & 1 & 1 & 1 & 6 & \\
\hline & $17.30-18.00$ & & & & & & & 0 & \\
\hline & $18.00-18.30$ & & & & & & & 0 & \\
\hline & $19.30-20.00$ & & & & & & & 0 & \\
\hline gesamt & & & & & & & & 6 Tage & 6 Tage \\
\hline \multirow[t]{4}{*}{ Person 2} & $17.00-17.30$ & 1 & 1 & 1 & 1 & 1 & & 5 & \\
\hline & $17.30-18.00$ & 1 & 1 & 1 & 1 & 1 & & 5 & \\
\hline & $18.00-18.30$ & 1 & 1 & 1 & 1 & 1 & & 5 & \\
\hline & $19.30-20.00$ & & & & & & 1 & 1 & \\
\hline gesamt & & & & & & & & 5 Tage & 6 Tage \\
\hline
\end{tabular}

$11 \mathrm{Um}$ das Beispiel konsistent halten zu können, muss die Person 2 zusätzlich zumindest an einem Werktag während der Woche von 19.30 - 20.00 Uhr die ARD oder das Nichtsehen bevorzugen. 


\subsubsection{Tagesablauf}

Kernstück der Media-Analysen sind die Abfragen zum stichtagsbezogenen Tagesablauf. Seit 1987 erfolgt dies in einer detaillierteren Form in Verbindung mit der Abfrage von Leittätigkeiten (Buß, 1998: 77 ff.). Sie werden seitdem für jede Viertelstunde zwischen 5 und 24 Uhr des letzten Tages vor dem Interview erhoben. Bei den aktuellen MA liegt der Schwerpunkt auf der Erfassung der Nutzung einzelner Radiosender. Von 1987 bis 1996 lag der Fokus ebenso auf der Ermittlung von senderspezifischen TV-Daten. Zusätzlich wird auch die Nutzung anderer Medien (Video, Schallplatten/Tonband/Kassetten/CD und seit 1997 PC) und von „Leittätigkeiten“ „zu Hause“ (Schlafen, Körperpflege, Essen, Haus-/Berufsarbeit, freie Zeit) und „außer Haus“ (im Auto unterwegs, Einkaufen, Berufsarbeit, Schule/Studium, Besuche, Kneipe) erhoben.

„Die Interviews werden nach Stichtagen gleichmäßig über die Woche verteilt, so dass in der Gesamtstichprobe die einzelnen Wochentage in etwa gleichem Umfang repräsentiert sind“ (Koschnick, 2004b). Der Interviewer unterstützt den Befragten in einem Gespräch, sich mit Hilfe der eben genannten Leittätigkeiten an den Verlauf des gestrigen Tages zu erinnern. Auf diese Weise wird nicht nur das Hören oder Sehen unterschiedlicher Sender einer Viertelstunde erfasst, sondern auch, welche Sender während welcher parallel stattfindenden Aktivität gehört oder gesehen wurden (Akinci, 2004: 2).

Codiert werden die Tagesablaufvariablen als sog. „Dummy“-Variablen, d. h. für jede Viertelstunde und jede Tätigkeit gibt es eine Variable ${ }^{12}$, welche die Information enthält, ob eine Tätigkeit ausgeführt wurde $(1=\mathrm{ja})$ oder nicht $(0=$ nein $)$.

Auch beim älteren MA-Erhebungsmodell von 1976 bis 1986 gab es Fragen zum jeweils gestrigen Tag (am Montag zu vorgestern, zum Sonntag wurde nicht befragt). Dieses „freie Gespräch“ beschränkte sich jedoch auf das Einholen von Nutzungswerten zu den elektronischen Medien Radio und Fernsehen, Leittätigkeiten im und außer Haus wurden (noch) nicht abgefragt. Ebenso wie bei der Abfrage zur Sehhäufigkeit pro Zeitabschnitt und Sender (siehe Abschnitt 2.1.3) konzentrierte sich die TV-Tagesablaufabfrage auf die werberelevante Zeit zwischen 17.00 und $20.00 \mathrm{Uhr}$, zusätzlich wurde die TV-Nutzung vor 17.00 Uhr pauschal abgefragt. In den Codeplänen wurde der Abfrageblock unter der Bezeichnung „gestern pro Zeitabschnitt und Sender“ aufgeführt. Viertelstundenweise wurden folgende Informationen pro TV-Sender erfasst: „1 = gestern ja“, „2 = gestern nein“ oder „3 = nicht WSK“ (Weitester Seherkreis). Eine längsschnittliche Nutzung ist demnach für 12 Viertelstunden von 1976 bis 1996 problemlos möglich.

\subsection{Pauschale Fernsehabfragen von 1976 bis beute}

Seit 1997 bzw. 2000 wird die Fernsehnutzung nicht mehr detailliert nach Sendern abgefragt (Keller \& Klingler, 1997), dennoch sind weiterhin Fernsehabfragen ${ }^{13}$ Teil des MAErhebungsprogramms. Allerdings beschränken sie sich auf die Abfrage einer pauschalen oder senderunspezifischen Fernsehnutzung. Erstens als Bestandteil der Tagesablaufabfrage, zweitens wird die generelle Sebhäufigkeit pro Zeitabschnitt abgefragt und drittens beinhaltet eine Freizeitskala auch Abfragen zur Mediennutzung.

12 In den Codeplänen sind die dazugehörigen Feldnummern zusammengefasst dargestellt. Beispiele für eine „Entschlüsselung“ befinden sich in der Tagesablaufdokumentation (Akinci, 2004).

13 Nahezu identische Abfragen gibt es auch zur Radionutzung. 


\subsection{1 (Pauschale) Fernsehnutzung im Tagesablauf}

Die in Abschnitt 2.1.4 vorgestellte Tagesablaufabfrage beschränkt sich seit 1997 auf eine Abfrage der pauschalen Fernsehnutzung innerhalb der MA Radio. Diese wird seit dem Jahr 2000 nicht mehr persönlich-mündlich, sondern telefonisch durchgeführt (Klingler \& Müller, 2000; Best \& Hagenah, 2006). Somit stehen für 76 Viertelstunden von $5.00 \mathrm{Ubr}$ bis $24.00 \mathrm{Ubr}$ Informationen zur pauschalen Fernsehnutzung im Tagesablauf von 1987 bis heute für längsschnittliche Sekundäranalysen zur Verfügung. Zwischen den Jahren 1976 bis 1986 beschränkte sich die „Tagesablaufabfrage“ auf die Zeiten zwischen 17.00 Uhr und $20.00 \mathrm{Uhr}$, so dass bei 12 Viertelstunden die pauschale Fernsehnutzung von 1976 bis heute untersucht werden kann.

\subsection{2 (Pauschale) Sebhäufigkeit pro Zeitabschnitt}

Von 1987 bis heute wird die pauschale Sehhäufigkeit pro Zeitabschnitt in einer normalen Woche ermittelt. Für insgesamt neun Zeitabschnitte ${ }^{14}$ wird abgefragt, an wie vielen der sechs Wochentage von Montag bis Samstag Fernsehen gesehen wird, so dass der „typische" Fernsehalltag ermittelt werden kann.

Die im CATI-Frageprogramm erscheinenden Intervieweranweisungen und Fragen sehen für die pauschale Sehbänfigkeit pro Zeitabschnitt seit 1987 bis heute wie folgt aus (ma_03_I_frb_cdb.pdf, S. 41):

Ich werde Ihnen jetzt einige Fragen zum Fernsehen stellen.

Denken Sie doch bitte einmal an die 6 Wochentage Montag bis Samstag.

An wie vielen von diesen 6 Wochentagen haben Sie in der letzten Zeit zwischen $6.00 \mathrm{Uhr}$ und 9.00 Uhr morgens Sendungen im Fernsehen gesehen?

INT.: Es geht um das Fernsehen, Anzahl der Tage eintragen!!

Sehe in dieser Zeit nie Fernsehen $>>0<$ eingeben

Und wie ist es Montags - Samstags in der Zeit zwischen [die nächsten unten stehenden Zeitabschnitte werden der Reihe nach eingeblendet]

Anzahl der Tage eintragen

09.00 Uhr und $13.00 \mathrm{Uhr}$

13.00 Uhr und $17.00 \mathrm{Uhr}$

17.00 Uhr und $18.00 \mathrm{Uhr}$

18.00 Uhr und $19.00 \mathrm{Uhr}$

19.00 Uhr und $20.00 \mathrm{Uhr}$

20.00 Uhr und $23.00 \mathrm{Uhr}$

23.00 Uhr und $02.00 \mathrm{Uhr}$

02.00 Uhr und $06.00 \mathrm{Uhr}$

Tage
Tage
Tage
Tage
Tage
Tage
Tage
Tage

14 Die Nutzungshäufigkeit wurde zu neun Zeitabschnitten (6-9, 9-13, 13-17, 17-18, 18-19, 19-20, 20-23, 23-2 und 2-6 Uhr) auf einer 7-stufigen Skala mit $0=$ „nie zu dieser Zeit“ bis $6=$, an 6 Wochentagen" abgefragt. 
Dieser Abfrageblock wurde prinzipiell auch von 1984 bis 1986 eingesetzt, also in den Jahren ohne Abfrage der senderspezifischen Sehhäufigkeit pro Tag und Zeitabschnitt. Allerdings beschränkte sich die Abfrage auf die Zeit zwischen 17.00 Uhr und 20.00 Uhr, die detaillierter in Halbstundenabschnitten erhoben wurde und dementsprechend in Stundendaten umkodiert werden müsste. Zusätzlich wurde ein zweistündiger Abschnitt „20.00 bis 22.00 Uhr“ abgefragt. Von 1976 bis 1983 wurde - wie in Abschnitt 2.1.3 genauer beschrieben - die senderspezifische Sehhäufigkeit pro Zeitabschnitt und Woche pro halbe Stunde zwischen 17.00 und 20.00 Uhr abgefragt und lässt sich mit Hilfe der Maximalwerte pro Sender annäherungsweise in eine Variable „pauschale Sehhäufigkeit pro Zeitabschnitt“ umkodieren.

\subsection{3 (Pauschale) Fernsebnutzung in der Freizeit}

In dem Abschnitt „Freizeitverhalten des Befragten“ lassen sich unter anderem Angaben zu medialen Tätigkeiten finden. Die Kernitems, wie Zeitungen lesen, Zeitschriften/Illustrierte lesen, Fernsehen und Radio hören, wurden seit 1984 durchgängig erhoben. Allerdings wurde die Skalierung geändert. Von 1993 bis heute liegt die Erhebungsspanne zwischen „1 = mehrmals in der Woche“ und „, $5=$ nie“.

Die im CATI-Frageprogramm erscheinenden Intervieweranweisungen und Fragen sehen für den Abfrageblock „Freizeitverhalten des Befragten“ von 1993 bis heute wie folgt aus (z. B. ma_03_I_frb_cdb.pdf, S. 40):

Ich lese Ihnen jetzt einige Tätigkeiten und Freizeitbeschäftigungen vor. Sagen Sie mir bitte zu jeder Tätigkeit, wie oft Sie dazu kommen, d. h. ist es „mehrmals in der Woche“, „mehrmals im Monat“, „etwa einmal im Monat“, „seltener“ oder „nie“?

Wie ist das mit ...

Kommen Sie dazu ... 1 = mehrmals in der Woche/ $2=$ mehrmals im Monat 3 = etwa einmal im Monat $/ 4=$ seltener $/ 5$ nie [zusätzlich codiert: 6 = keine Angabe; $d$. Verf.]

Fernsehen/Radio hören/ Schallplatten, CDs, Kassetten, Tonband hören/ Video-Kassetten ansehen/ ins Kino gehen/ in Theater, Konzert, kulturelle Veranstaltungen gehen/ Zeitung lesen/ Zeitschriften, Illustrierte lesen/ Bücher lesen/ Basteln, Heimwerken, Schneidern, Stricken/ Sport treiben, sich trimmen/ Ausgehen: Restaurant, Gaststätte, Kneipe, Disco

Zwischen 1984 und 1992 beinhaltete die Skala Ausprägungen zwischen „1 = täglich/ fast täglich“ und „6 = nie/ so gut wie nie“. Für eine longitudinale Nutzung von 1984 bis heute könnten die Daten der älteren Abfrage in das Format der aktuellen Abfrage umkodiert werden, allerdings unter Inkaufnahme eines Informationsverlustes und eines zu erwartenden Methodeneffektes (ausführlicher für die identisch abgefragte Variable zur Sportaktivität: Hagenah, 2003). Die Mediennutzung innerhalb des Abfrageblockes Freizeitaktivität wäre ein ideales Instrument für intermediale Vergleiche und wäre darüber hinaus für eine Einordnung der Mediennutzung im Vergleich zu (anderen) Freizeitaktivitäten zu gebrauchen. Sie wurde jedoch nur mit einer groben Skalierung (5 Stufen von „nie“ bis „mehrmals in der Woche“) erfasst, so dass bei Radio, Fernsehen und Tageszeitung die häufige Nutzung nicht differenziert werden kann, und der Informationsgehalt demzufolge gering ist. Dieser Abfrageblock ist eher für nicht täglich verfolgte Freizeitaktivitäten - wie Ausgehen oder Sport treiben - angemessen. Lediglich die Zeitschriftenabfrage enthält brauchbar differenzierende Informationen. Nutzbar sind die Freizeitdaten - unter Berücksichtigung des Methodeneffektes - von 1984 bis heute. 


\subsection{Zusammenfassung: Senderspezische und pauschale Fernsehabfragen}

In Tabelle 2 werden die longitudinalen Nutzungsmöglichkeiten der senderspezifischen und pauschalen Fernsehabfragen zusammenfassend miteinander verglichen. Bei der bis 1999 eingesetzten senderspezifischen Fernsehabfrage lassen sich Informationen zum Generalfilter von 1976 bis 1999 und zum Zeitfilter von 1976 bis 1996 problemlos miteinander verknüpfen. Dies gilt auch für die Daten zur Fernsehnutzung im Tagesablauf von 1976 bis 1996, allerdings beschränkte sich die Erhebung bis 1986 auf die werberelevante Zeit zwischen 17.00 und $20.00 \mathrm{Uhr}$. Informationen zum kompletten, senderspezifisch erbobenen Tagesablauf zwischen 05.00 und 24.00 Uhr liegen nur für den Zeitraum von 1987 bis 1996 vor. Kritischer sind die longitudinalen Nutzungsmöglichkeiten der Frequenzvariablen einzuschätzen, da sie methodisch mit einem sich ändernden Ansatz (1976-1983: Sehhäufigkeit pro Zeitabschnitt und Sender vs. 1987 - 1999: Sehhäufigkeit pro Sender; siehe Abschnitt 2.1.3) und zudem im alten Modell lediglich für die Zeit von 17.00-20.00 Uhr erhoben wurden. Allerdings können auch sie unter Beachtung der zu erwartenden Methodeneffekte für longitudinale Analysen von 1976 bis 1999 genutzt werden.

Längere Zeitreihen lassen sich für die bis dato erhobene pauschale Fernsebabfrage bilden. Für die Zeit zwischen 17.00 und $20.00 \mathrm{Uhr}$ - also für 12 Viertelstunden - können Informationen zur pauschalen Fernsehnutzung im Tagesablauf von 1976 bis heute problemlos miteinander verknüpft werden. Nach einer Umkodierung der Variablen von 1984 bis 1992 können grobe Daten zur Fernsehnutzung in der Freizeit von 1984 bis heute - unter Berücksichtigung eines Methodeneffektes - genutzt werden. Methodisch bedenklicher ist die longitudinale Nutzung der pauschalen Frequenzvariablen pro Woche einzuschätzen, da sie methodisch mit einem sich ändernden Ansatz (1976 - 1983: Sehhäufigkeit pro Zeitabschnitt vs. 1987 - heute: Sehhäufigkeit) und zudem im alten Modell lediglich für die Zeit von 17.00-20.00 Uhr erhoben wurden. Allerdings kann auch sie unter Beachtung der zu erwartenden Methodeneffekte für longitudinale Analysen von 1976 bis heute genutzt werden.

\section{Fazit}

Wie die Dokumentenanalyse gezeigt hat, lassen sich aus den Media-Analysen lange Zeitreihen erstellen, welche die Fernsehrezeption von 1976 bis 1999 senderspezifisch und von 1976 bis heute pauschal nachzeichnen können.

Ziel dieses Beitrags war es, die Möglichkeiten der Zeitreihenbildung mit Media-Analysen transparent für andere nachvollziehbar darzustellen, auch um somit der Forderung von Lauf und Peiser (1999: $231 \mathrm{ff}$.) nach einer ausführlichen Methodendokumentation bei der Aufbereitung von Datensätzen zu folgen. In einem nächsten Schritt sollen die Zeitreihen auch gebildet werden. Dafür erscheint es sinnvoll, analog wie auch für die Radiodaten konstatiert (Hagenah, 2006), ergänzende soziodemographische Aufgliederungen vorzunehmen.

Darüber hinaus sollten ähnliche Dokumentenanalysen die Zeitschriften- und Zeitungsvariablen der Media- und Leser-Analyse zum Untersuchungsgegenstand haben, da diese inhaltlich ein ähnliches und zeitlich ein darüber hinaus gehendes Longitudinalpotenzial für Sekundäranalysen von 1954 bis heute aufweisen.

Mit Blick auf die Inhalte der MA lassen sich - ähnlich wie beim Wechsel vom persönlichen zum telefonischen Interview (ausführlicher bei Best \& Hagenah, 2006; 2006a) - Erhebungseffekte an den methodologischen Umbruchstellen vermuten und näher un- 


\begin{tabular}{|c|c|c|c|c|}
\hline 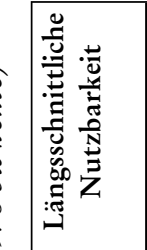 & 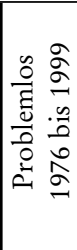 & 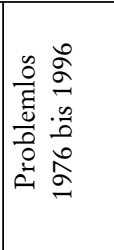 & 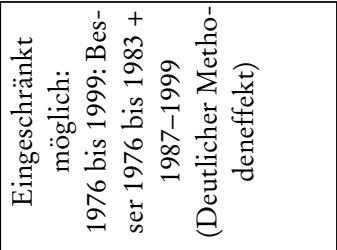 & 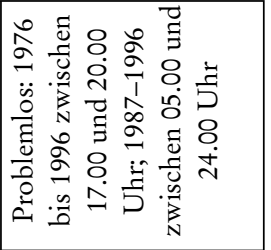 \\
\hline 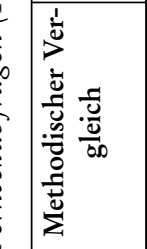 & 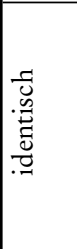 & 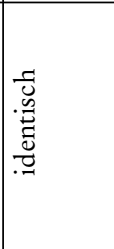 & 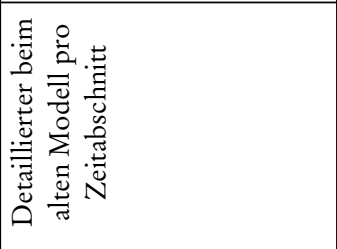 & 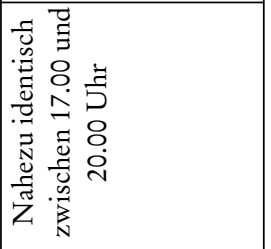 \\
\hline 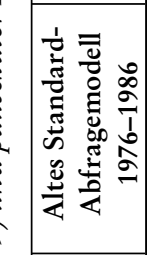 & & 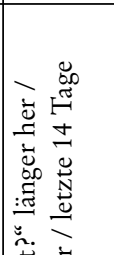 & 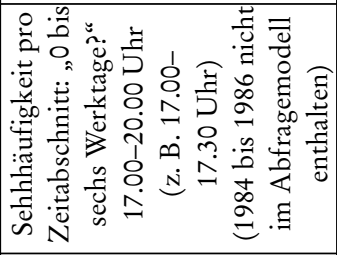 & 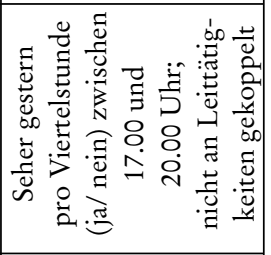 \\
\hline 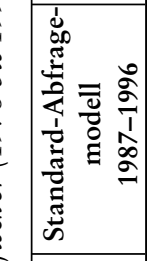 & 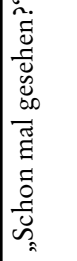 & 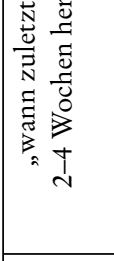 & 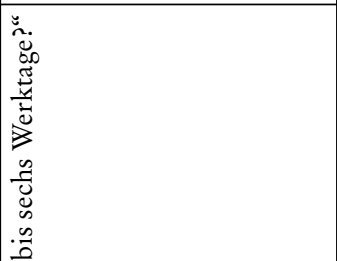 & 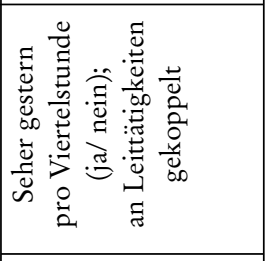 \\
\hline 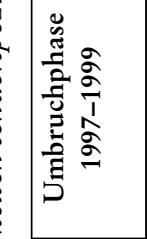 & & 1 & 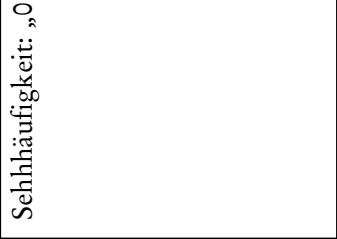 & 1 \\
\hline 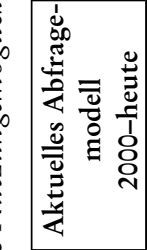 & 1 & 1 & 1 & 1 \\
\hline 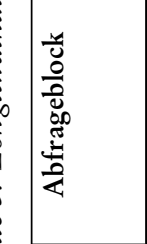 & 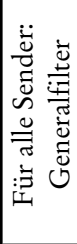 & 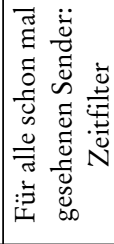 & 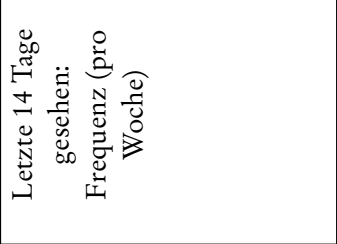 & 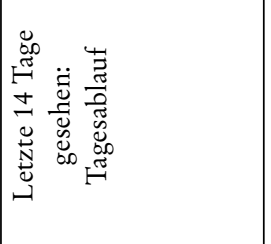 \\
\hline & \multicolumn{4}{|c|}{ 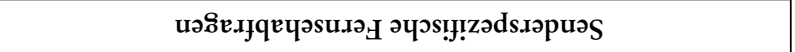 } \\
\hline
\end{tabular}




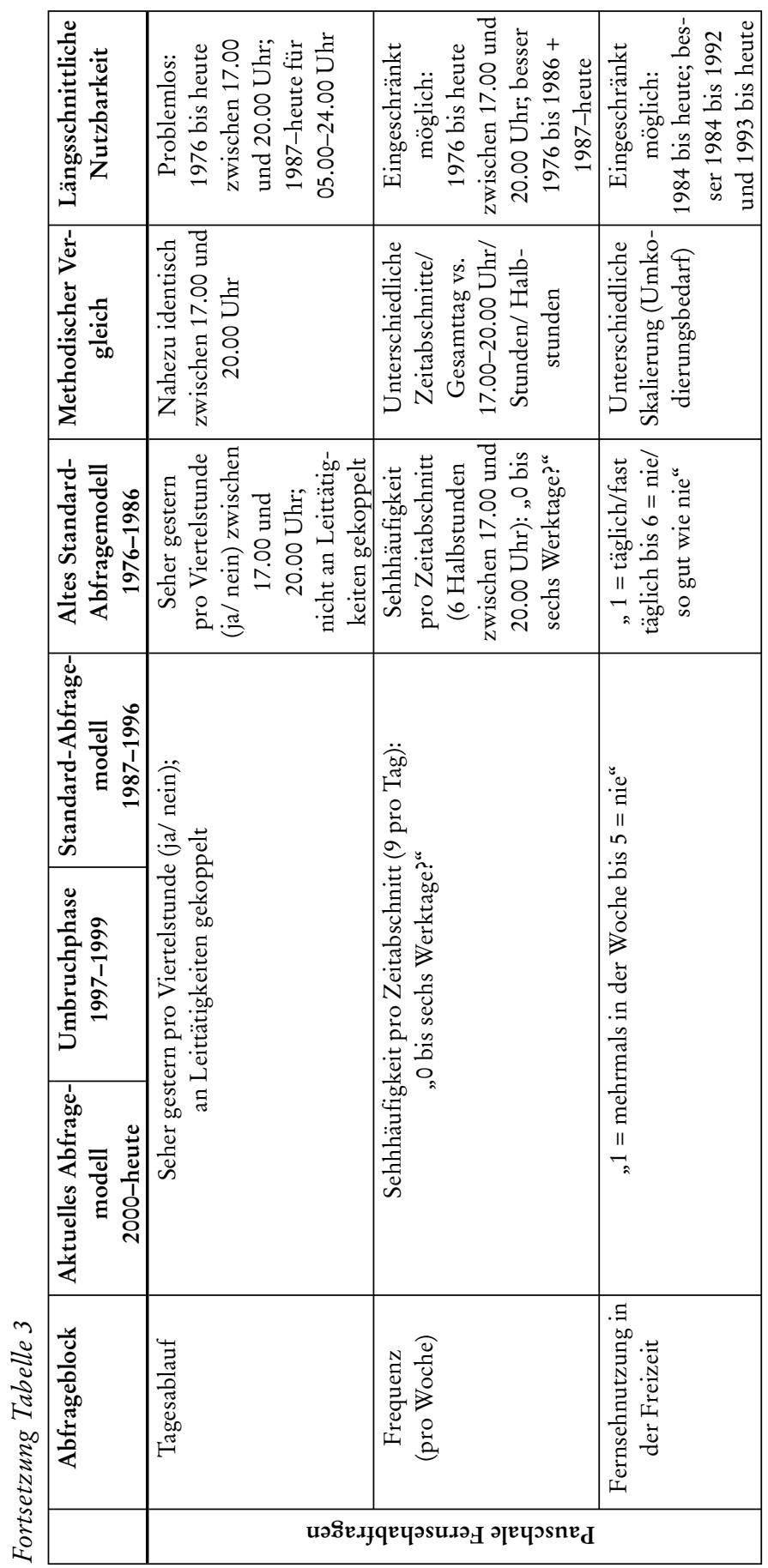


tersuchen. Entsprechende Ergebnis „sprünge“ sollten bei der Zeitreihenbildung beachtet und ggf. auch graphisch markiert werden.

Letztlich erscheint es sinnvoll, Standards der Erhebungs- und ggf. auch der Auswertungsdokumentation zur Erleichterung von Replikationen im Sinne einer intersubjektiv nachvollziehbaren wissenschaftlichen Herangehensweise zu diskutieren. Entsprechende Dokumentationen und Aufbereitungen könnten auch für andere im Zentralarchiv für empirische Sozialforschung in Köln lagernde kommunikations- oder sozialwissenschaftliche Datensätze, wie die Langzeitstudie Massenkommunikation oder die Typologie der Wünsche (Lauf, 2002: 253), erstellt werden, um die Sekundärnutzungen zu erleichtern.

\section{Literatur}

Akinci, H. (2004): Leitfaden für den Umgang mit Tagesablaufdaten. http://www.wiso.uni-koeln. de/medien/download/tagesablaufdokumentation.pdf, erstellt am 28.6.04 [1.11.2006].

Best, H. \& Hagenah, J. (2006): Vom persönlichen zum telefonischen Interview: Probleme der Stichproben-Zusammensetzung und des Antwortverhaltens. In: Hagenah, J. \& Meulemann, H. (Hrsg.), Sozialer Wandel und Mediennutzung in der Bundesrepublik Deutschland (S. 35-56). LIT Verlag: Münster.

Best, H. \& Hagenah, J. (2006a). Die Tagesablauf-Abfrage der Media-Analyse von 1975 bis heute. In: Wirth, W. \& Matthes, J. (Hrsg.), Die Brücke zwischen Theorie und Empirie: Operationalisierung, Messung und Validierung in der Kommunikationswissenschaft: Abstracts (S. 58-63). IPMZ: Zürich.

Bilandzic, H. (1998): Formale Merkmale individueller Fernsehnutzung. In: Klingler, W., Roters, G. \& Zöllner, O. (Hrsg.), Fernsehforschung in Deutschland: Themen - Akteure - Methoden: Band 2 (S. 744-763). Baden-Baden: Nomos Verlagsgesellschaft.

Bortz, J. \& Döring, N. (2002): Forschungsmethoden und Evaluation. Springer: Heidelberg.

Buß, M. (1998): Leistungsfähigkeit und Grenzen der Media Analyse. In: Lindner-Braun, C. (Hrsg.), Radioforschung - Konzepte, Instrumente und Ergebnisse aus der Praxis (S. 77-82). Opladen: Westdeutscher Verlag.

Diekmann, A. (2004): Empirische Sozialforschung. Reinbek bei Hamburg: Rowohlt Verlag.

Hagenah, J. (2003): ma-Beispielanalyse „Sportaktivität“. http://www.wiso.uni-koeln.de/medien/ download/ma_analysebeispiel_sportaktivitaet.pdf, erstellt am 19.9.2003 [1.11.2006].

Hagenah, J. (2006): Möglichkeiten der Nutzung von Media-Analyse Radiodaten für Sekundäranalysen von 1972 bis heute. Medien E Kommunikationswissenschaft, 3, 457-485.

Karnowski, V. (2003): Von den Simpsons zur Rundschau. Wie sich Fernsebnutzung im Laufe des Lebens verändert. Eine Sekundäranalyse von Daten aus dem AGF/GfK-Fernsehpanel. München: Verlag Reinhard Fischer.

Keller, M. \& Klingler, W. (1997): Die Hörfunknutzung legt vor allem im Westen deutlich zu. Media Perspektiven, 10/1997, 526-536.

Klingler, W. \& Müller, M. (2000): MA 2000 Radio: Erstmals mit Telefoninterviews erhoben. Media Perspektiven, 9/2000, 414-426.

Koschnick, W. J. (2004a): Filterfrage (Filterführung, Screening). In: W. J. Koschnick (Hrsg.), FocusLexikon Werbeplanung - Mediaplanung - Marktforschung - Kommunikationsforschung - Mediaforschung. http://www.medialine.de (nach kostenloser Registrierung: Wissen, Medialexikon, Stichwort: Filterfrage) [3.11.2006].

Koschnick, W. J. (2004b): Abfrage (Medienabfrage). In: W. J. Koschnick (Hrsg.), Focus-Lexikon Werbeplanung- Mediaplanung Marktforschung-Kommunikationsforschung-Mediaforschung. http://www.medialine.de (nach kostenloser Registrierung: Wissen, Medialexikon, Stichwort: Abfrage) [3.11.2006].

Lauf, E. \& Peiser, W. (1999): Validität der Studie Massenkommunikation. Rundfunk und Fernseben, 47/ 2, 231-242.

Lauf, E. (2002): Freiheit für die Daten! Sekundäranalysen und Datenbestände in der deutschen 
Medien- und Kommunikationswissenschaft. Medien E Kommunikationswissenschaft, 50 (2), 247-260.

Hagenah, J., Meulemann, H. \& Akinci, H. (2006): European Data Watch: German Media-Analyse (MA): A large scale commercial data source available for secondary analyses on media use and social change. Schmollers Jabrbuch. Journal of Applied Social Science Studies / Zeitschrift für Wirtschafts- und Sozialwissenschaften, 1, 129-137.

Mai, L. (2003): Die Media-Analyse Radio. Eine Methodenbeschreibung. Frankfurt: ARD-Werbung SALES \& SERVIES GmbH Corporate Communication.

Müller, D. K. \& Mai, L. (2006): Das Erhebungsmodell der Media-Analyse Radio. In: Hagenah, J. \& Meulemann, H. (Hrsg.), Sozialer Wandel und Mediennutzung in der Bundesrepublik Deutschland (S. 18-34). LIT Verlag: Münster.

Müller, D. K. (2003): Methodentest 1995. Modelle zur Erhebung der Radionutzung in der mediaanalyse. In: Müller, D. K. \& Wiegand, J. (Hrsg.), Von face-to-face zu CATI: Dokumentation der Experimente und Methoden zur Optimierung der media-analyse-Radio. Band 18 (S. 33-56). AG.MA: Frankfurt.

Scheler, H.-E. (1983): Experimente, ihr Anlaß und ihr Ergebnis. Vortrag auf der AG.MA-Mitgliederversammlung am 23. November 1983. In: Arbeitsgemeinschaft Media-Analyse (Hrsg.), Dokumentation der Experimente 1976-1983 zur Veränderung des Fragebogens der MA AG. MA Schriften Band 10 (S. 387-393). AG.MA: Frankfurt.

www.agf.de (2006): Arbeitsgemeinschaft Fernsebforschung (AGF), http://www.agf.de/ [1.11.2006].

www.gfk.de (2006). Fernsehzuschauerforschung in Deutschland, www.gfk.de/fernsehforschung [1.11.2006]. 